\title{
Simulations of emerging flux in a coronal hole: oscillatory reconnection
}

\author{
M. J. Murray ${ }^{1}$, L. van Driel-Gesztelyi ${ }^{1,2,3}$, and D. Baker ${ }^{1}$ \\ 1 University College London, Mullard Space Science Laboratory, Holmbury St Mary, Dorking, Surrey, RH5 6NT, UK \\ e-mail: mjm@mssl.ucl.ac.uk \\ 2 Observatoire de Paris, LESIA, UMR 8109 (CNRS), Meudon-Principal Cedex, France \\ 3 Konkoly Observatory, Budapest, Hungary
}

Received 17 June 2008 / Accepted 27 November 2008

\begin{abstract}
Context. Observations and simulations show that reconnection will take place when a flux tube emerges into a coronal hole, which is characterised by magnetic fieldlines "open" towards interplanetary space. Although the mechanism by which reconnection is initiated has been thoroughly studied, the long-term evolution of this reconnecting magnetic system remains unreported.

Aims. We aim to understand the long-term evolution of the reconnecting flux tube and coronal hole system and, in particular, to ascertain whether it can reach an equilibrium state in which all reconnection has ceased. By determining the evolution in this particular scenario, we aim to be able to select a subset from the broad spectrum of reconnecting systems, which will undergo the same progression to equilibrium.

Methods. Using a 2.5-dimensional numerical magnetohydrodynamic (MHD) code, we evolve a simple stratified atmospheric domain, which is endowed with a vertical magnetic field, representing the interior of a coronal hole, and a horizontal buoyant flux tube that is placed near the bottom of the domain. To investigate the long-term evolution of the system, we continue to study the domain long after the flux tube has emerged and reconnection has commenced between the magnetic fields of the flux tube and coronal hole.

Results. We find that a series of reconnection reversals (or oscillatory reconnection) takes place, whereby reconnection occurs in distinct bursts and the inflow and outflow magnetic fields of one burst of reconnection become the outflow and inflow fields in the following burst of reconnection, respectively. During each burst of reconnection the gas pressure in the bounded outflow regions increases above the level of that in the inflow regions and, eventually, gives rise to a reconnection reversal. In consecutive bursts of reconnection, the contrast in the gas pressure across the boundaries of the inflow and outflow regions decreases and, over time, the system settles towards equilibrium. Once the equilibrium state is reached, all reconnection ceases. This is the first reported instance of oscillatory reconnection initiated in a self-consistent manner, and the signatures of the mechanism compare favourably with observations of select flux emergence events and with solar and stellar flares.

Conclusions. Across the broader spectrum of reconnecting systems, oscillatory reconnection will only occur if the outflow regions are quasi-bounded during each burst of reconnection. The swaying outflow jet and periodic heating signatures of oscillatory reconnection are exceedingly similar to those exhibited by MHD modes and, in many observations, distinction between the two mechanisms may be impossible.
\end{abstract}

Key words. magnetohydrodynamics (MHD) - methods: numerical - Sun: magnetic fields - Sun: atmosphere - Sun: flares Sun: oscillations

\section{Introduction}

Magnetic fields are continually emerging on the Sun, across its entire surface and on a range of scales. The configuration of the pre-existing, surrounding atmospheric field plays a key role in determining the evolution of the emerging field. Magnetic reconnection will readily set in given appropriate conditions, releasing magnetic energy stored in any stressed part of the fields and enabling the fields to relax to lower energy states. The topology of the magnetic fields will be modified during the reconnection process, with heating and jets generated as by-products.

The dynamic onset of reconnection as a result of newly emerged flux has already been thoroughly studied (Shibata et al. 1992; Yokoyama \& Shibata 1995, 1996; Miyagoshi \& Yokoyama 2003, 2004; Isobe et al. 2005, 2006; Archontis et al. 2004, 2005, 2006, 2007; Galsgaard et al. 2005, 2007; Moreno-Insertis et al. 2008). It is reasonable to assume that, in many cases, reconnection cannot continue indefinitely but the subsequent relaxation of the system and the conclusion of reconnection remains unreported to date.

In this paper, we consider the example of flux emerging into a coronal hole. Previous numerical simulations of such a scenario reveal that reconnection readily sets in between the emerging flux and pre-existing coronal hole field, which is characterised by magnetic fieldlines "open" towards interplanetary space (Yokoyama \& Shibata 1995, 1996; Moreno-Insertis et al. 2008). Using numerical simulations, we follow the long-term evolution of the system with the aim of understanding how the reconnecting magnetic system reaches an equilibrium state in which all reconnection has ceased. Although this is a specific example of reconnection initiated by flux emergence, we believe we will be able to understand how a broader selection of reconnecting systems will progress towards equilibrium once the evolution has been determined for this case.

The paper is laid out as follows: Sect. 2 introduces the setup of the domain and the numerical code employed. Section 3 
(a)

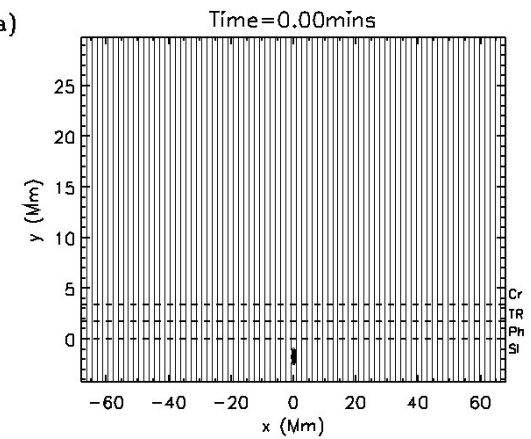

(b)

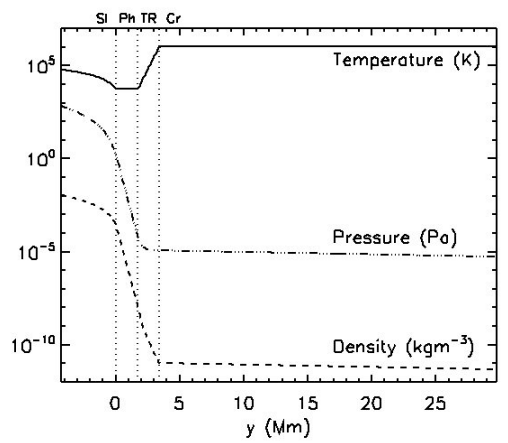

Fig. 1. Initial conditions of the domain: a) fieldlines of the flux tube and the domain filling vertical magnetic field of the coronal hole and b) temperature (solid), gas pressure (triple dot-dashed) and density (dashed) profiles in height. The solar interior $(\mathrm{SI})$, photosphere $(\mathrm{Ph})$, transition region (TR) and corona $(\mathrm{Cr})$ are indicated by horizontal dashed and vertical dotted lines in a) and $\mathbf{b})$, respectively. presents the results of the simulations, describing the mechanism by which the reconnecting system reaches equilibrium (3.1 and 3.2) and the observational signatures associated with it (3.3). Section 4 will consider the validity of the model and observational evidence of this relaxation mechanism at work in real scenarios. Finally, Sect. 5 summarises our findings, highlighting their importance in a broader solar context.

\section{Numerical method and domain setup}

The numerical results presented in this paper were obtained using the Lagrangian remap scheme Lare2d (Arber et al. 2001). This code has previously been used by Leake \& Arber (2006) to investigate the effects of partial ionisation and Newton cooling during flux emergence and by Archontis et al. (2007) to study the emergence of two interacting flux tubes. In the 2.5-dimensional domain, $y$ is the vertical coordinate and $z$ is the horizontal coordinate in which all quantities have spatial partial derivatives equal to zero. The evolution of the simulation domain is governed by the following time-dependent, resistive, dimensionless MHD equations:

$$
\begin{aligned}
\frac{\partial \rho}{\partial t} & =-\nabla \cdot(\rho \boldsymbol{v}), \\
\frac{\partial \rho \boldsymbol{v}}{\partial t} & =-\nabla \cdot(\rho \boldsymbol{v} \boldsymbol{v})+(\nabla \times \boldsymbol{B}) \times \boldsymbol{B}-\nabla p+\rho \boldsymbol{g}, \\
\frac{\partial \boldsymbol{B}}{\partial t} & =\nabla \times(\boldsymbol{v} \times \boldsymbol{B})+\eta \nabla^{2} \boldsymbol{B}, \\
\frac{\partial \rho \epsilon}{\partial t} & =-\nabla \cdot(\rho \boldsymbol{\epsilon} \boldsymbol{v})-p(\nabla \cdot \boldsymbol{v})+Q_{\text {joule }}+Q_{\text {shock }},
\end{aligned}
$$

with density $\rho$, time $t$, velocity $\boldsymbol{v}$, magnetic field $\boldsymbol{B}$, gas pressure $p$, acceleration due to gravity $\boldsymbol{g}=-g \boldsymbol{y}$, resistivity $\eta$, specific energy density $\epsilon=p /(\gamma-1) \rho$, joule heating $Q_{\text {joule }}$ and viscous heating at shocks $Q_{\text {shock}}$. The ratio of specific heats, gravitational acceleration and resistivity are taken to be constants with dimensionless values $\gamma=5 / 3, g=1.0$ and $\eta=0.001$, respectively. Heat conduction and radiative effects are neglected.

The values retuned from (1)-(4) are made dimensional using the following solar photospheric values: pressure scale height $H_{\mathrm{ph}}=1.7 \times 10^{5} \mathrm{~m}$, time $t_{\mathrm{ph}}=25.0 \mathrm{~s}$, velocity $v_{\mathrm{ph}}=6.8 \times$ $10^{3} \mathrm{~m} \mathrm{~s}^{-1}$, density $\rho_{\mathrm{ph}}=3.0 \times 10^{-4} \mathrm{~kg} \mathrm{~m}^{-3}$, pressure $p_{\mathrm{ph}}=$ $1.2 \times 10^{4} \mathrm{~Pa}$, temperature $T_{\mathrm{ph}}=5.6 \times 10^{3} \mathrm{~K}$, magnetic field $B_{\mathrm{ph}}=$ $1.3 \times 10^{3} \mathrm{G}$, surface gravity $g_{\mathrm{ph}}=2.7 \times 10^{2} \mathrm{~m} \mathrm{~s}^{-1}$ and mean atomic weight $\tilde{\mu}=1.0$. All values discussed within the remainder of this paper will be dimensional unless otherwise stated.

The numerical domain comprises four horizontal layers, as shown in Fig. 1a. Moving upwards from the bottom boundary of the domain there is a solar interior that is marginally stable to convection, a $5600 \mathrm{~K}$ isothermal photosphere, a transition region with rapid temperature increase and a $1.1 \mathrm{MK}$ isothermal corona. Each of the regions is initially set in hydrostatic equilibrium and the resulting gas pressure and density profiles, together with temperature, can be seen in Fig. $1 \mathrm{~b}$.

The domain is endowed with a negatively directed vertical magnetic field of $19.5 \mathrm{G}$, representing the interior of a unipolar coronal hole. The coronal temperature and density within the coronal hole are in line with the findings of Zhang et al. (2007) and the field strength is taken from Baker et al. (2009).

In addition, a magnetic flux tube is inserted into the solar interior at a depth of $-1.7 \mathrm{Mm}$ and centred about $x=0.0 \mathrm{Mm}$. The magnetic field of the flux tube is prescribed in the cylindrical coordinate system $(r, \theta, z)$ by $\boldsymbol{B}=\left(0, \alpha r B_{0} \mathrm{e}^{-r^{2} / R^{2}}, B_{0} \mathrm{e}^{-r^{2} / R^{2}}\right)$. The maximum field strength of the tube is given by $B_{0}=3.25 \times 10^{3} \mathrm{G}$, the radius at which the axial field strength falls by a factor of $1 / e$ is set at $R=4.25 \times 10^{5} \mathrm{~m}$ and the degree of rotation of the fieldlines about the axis is taken as $\alpha=-0.064 \times 2 \pi$ for each $1.7 \times 10^{5} \mathrm{~m}$ length in the axial direction. The tube is set in radial force balance and thermal equilibrium with the external plasma. Hence, a difference in density exists such that the tube is buoyant in comparison to its surroundings and will rise bodily towards the surface at the start of the simulations.

The simulation domain models the region $-68.0 \mathrm{Mm} \leq x \leq$ 68.0 Mm and $-4.25 \mathrm{Mm} \leq y \leq 29.75 \mathrm{Mm}$ using $1600 \times 400$ gridpoints of uniform spacing. The boundaries are closed in $x$ and $y$ and no damping zones are imposed. Convergence testing has been carried out in a domain with $800 \times 200$ gridpoints and in a domain measuring $63.75 \mathrm{Mm}$ above the solar surface.

\section{Results}

The rise and emergence of the flux tube are well documented in existing literature and the reader is directed to Murray et al. (2006) and references therein for details. As observed in previous simulations of flux emergence in a coronal hole, the emerging magnetic field compresses the neighbouring coronal hole field as it expands vertically and horizontally into the atmosphere. In the left part of the loop system, the emerged field is directed positively out of the solar surface and the adjacent coronal hole field is oppositely directed. Hence, a current sheet builds at the left-hand side interface between the two flux systems, as shown in Fig. 2a. Reconnection, depicted in Fig. 2b, commences 41.6 min into the simulations and we continue to study the system for a further $1.5 \mathrm{~h}$.

We find oscillatory reconnection sets in as the system searches for equilibrium. In Sect. 3.1, we discuss the meaning of oscillatory reconnection and provide evidence for it occurring in our simulations. In Sect. 3.2 we discuss the mechanism that drives this oscillatory reconnection and, finally, in Sect. 3.3 we illustrate the consequences of oscillatory reconnection. 
(a)

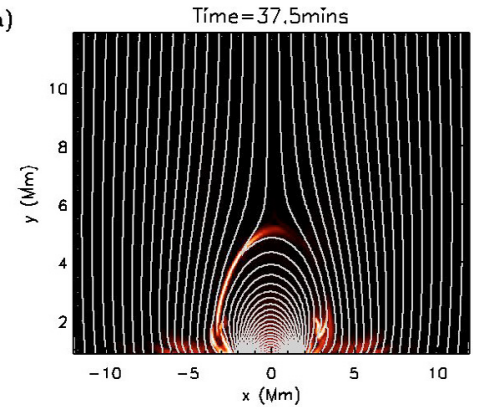

(b)

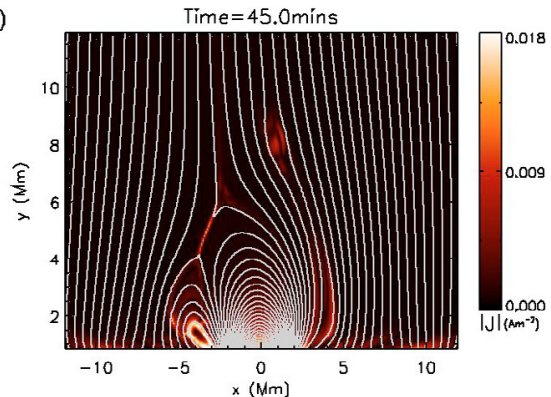

Fig. 2. Contours of current density, $|\boldsymbol{J}|$, overlaid with a selection of fieldlines after a) $37.5 \mathrm{~min}$ and b) $45.0 \mathrm{~min}$.
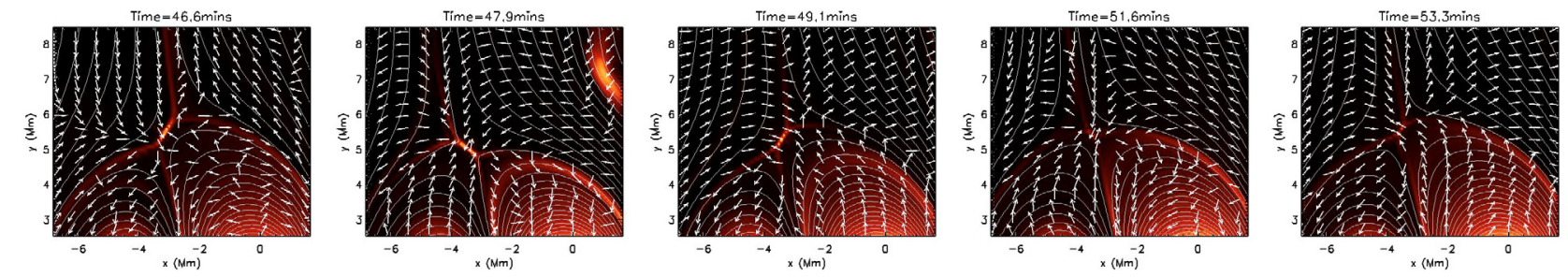

Fig. 3. Contours of current density, $|\boldsymbol{J}|$, overlaid with velocity arrows of unit length. The images illustrate reconnection of types $R_{1}$ and $R_{2}$, starting with $R_{1}$ and alternating from left to right.

\subsection{Evidence of oscillatory reconnection}

The reconnection that is first initiated, illustrated in Fig. 3 at $46.6 \mathrm{~min}$, we classify as being of type $R_{1}$. Inflows acting perpendicular to the current sheet bring the emerged loops and open field with footpoints at the left-hand side of the emerged loops (hereafter open left field) into the diffusion region. In the 2.5-dimensional simulations the reconnection is essentially twodimensional, whereby an input pair of fieldlines are "cut" and "glued" to produce a pair of new output fieldlines. Here, the new output fieldlines are a closed loop, situated between the emerged loops and open left field, and a new open fieldline whose footpoint passes to the right of the emerged loops (hereafter open right field). New magnetic field is carried into the outflow regions by flows emanating from the ends of the current sheet. Reconnection of this type, which maintains the quantity of open field, is specifically termed interchange reconnection (Crooker et al. 2002; Baker et al. 2007).

Reconnection of type $R_{1}$ lasts for $5.4 \mathrm{~min}$ and is followed by reconnection of type $R_{2}$, shown in Fig. 3 at $47.9 \mathrm{~min}$. In this new reconnection phase, the current sheet lies at an angle of $\sim 45^{\circ}$ anticlockwise from the vertical, between the new closed loops and the open right field. The inflows to and outflows from the current sheet have a reversed direction from $R_{1}$. Now, the inflow field is the product of the reconnection in $R_{1}$ and the outflow field replenishes the emerged loops and original open left field.

We refer to an interval of time that consists of a single type of reconnection as a reconnection phase and a change in the type of reconnection $\left(R_{1}\right.$ to $R_{2}$ or $R_{2}$ to $\left.R_{1}\right)$ as a reconnection reversal. As shown in Fig. 3 , we find a series of reconnection reversals, with individual phases of reconnection lasting between $1.5 \mathrm{~min}$ and $32.1 \mathrm{~min}$. Following conventions in previous literature (Craig \& McClymont 1991), we use the terminology oscillatory reconnection to refer to the overall reconnection process encompassing this series of reconnection reversals.

We quantitatively investigate the reconnection by considering the changing connectivity of the open flux over time. We trace 600 equally spaced fieldlines from the top boundary of the domain, over the region $-8.5 \mathrm{Mm} \leq x \leq 8.5 \mathrm{Mm}$. At each time step we calculate whether individual fieldlines pass to the left or right of the emerged loop system as they enter the solar

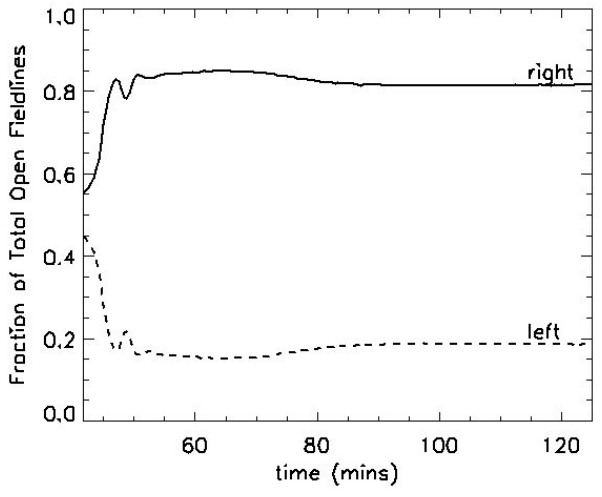

Fig. 4. Open fieldlines passing to the left (dashed) and right (solid) of the emerged loops over time as a fraction of the total number of the open fieldlines traced.

interior. Any change in the number of fieldlines passing to each side indicates reconnection has occurred: increases in fieldlines to the right result from reconnection of type $R_{1}$ and increases in fieldlines to the left result from reconnection of type $R_{2}$.

Figure 4 shows the change in the open fieldlines passing to each side of the emerged loops over time as a fraction of the total number of fieldlines traced. Passing to the left and passing to the right are mutually exclusive and exhaustive events for each traced fieldline and, therefore, any change in the fraction of fieldlines passing to the left is matched by an equal and opposite change in the fraction of fieldlines passing to the right. The reconnection reversals are recognisable as peaks (troughs) for an $R_{1}$ to $R_{2}$ reversal and troughs (peaks) for an $R_{2}$ to $R_{1}$ reversal in the right (left) fraction. From Fig. 4 , it is possible to distinguish a total of five reconnection reversals.

The largest fraction of the fieldlines, $27 \%$, change topology during the first reconnection phase and much smaller fractions are converted in the subsequent phases. Hence, flux reconnected early in the first $R_{1}$ phase maintains its new topology for the remainder of the simulations but flux reconnecting late in this first phase may change its footpoint location (left or right) with each new reversal. The final reconnection phase, 65-90 $\mathrm{min}$, is 
(a)

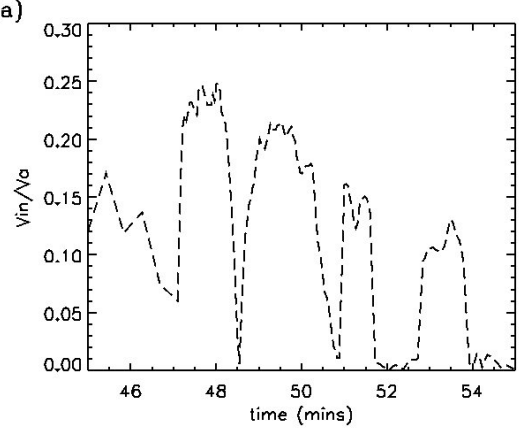

(b)

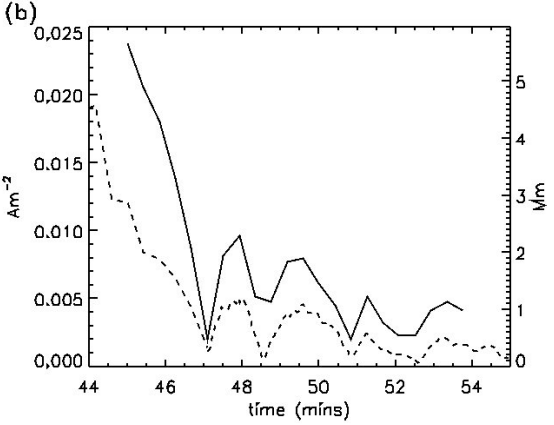

Fig. 5. a) Reconnection rate given by the inflow Alfvén Mach number and b) maximum current in the current sheet (dashed), measured in units of $\mathrm{Am}^{-2}$, and length of the current sheet (solid), measured in units of $\mathrm{Mm}$, as functions of time. the longest phase but only $3 \%$ of fieldlines change connectivity. From 90 min onwards, no further reconnection occurs.

We evaluate the reconnection to a higher degree of accuracy by calculating the reconnection rate defined by the inflow Alfvén Mach number

$M_{\text {in }}=v_{\text {in }} / v_{\mathrm{a}}$,

where $v_{\text {in }}$ is the inflow velocity towards the diffusion region and $v_{\mathrm{a}}$ is the Alfvén velocity measured at the location of the inflow velocity. Figure 5a shows that during each reconnection phase the rate of reconnection increases to a peak value before decreasing prior to the next reversal. In each new phase, the peak reconnection rate is smaller than that of the previous phase and suggests the system is settling towards a state of equilibrium. This assertion will be considered further in Sect. 3.2, where the mechanism driving the oscillatory reconnection is described.

The use of the Alfvén Mach number as a proxy for the reconnection rate is valid under the assumption of a steady state regime, in which the plasma carries the fieldlines into the diffusion layer at the same rate as the fieldlines are trying to diffuse out (Priest \& Forbes 2000). Under this regime, the Alfvén Mach number is proportional to $\eta|\boldsymbol{J}|$ in the diffusion region and we find that this is indeed the case in our simulations, as shown in Fig. 5b. The maximum current in the current sheet exhibits an increase and a decrease during each reconnection phase and a general trend of decreasing peak values during consecutive reconnection phases. In addition, we find that the length of the current sheet is also proportional to the reconnection rate and the maximum current. The synchronised evolution of these three quantities will be discussed further in Sect. 3.2.

\subsection{Driving mechanism for oscillatory reconnection}

In order to determine the driving force of the oscillatory reconnection process, we begin by investigating why the first bout of $R_{1}$ reconnection ceases and reconnection of type $R_{2}$ sets in.

As discussed in Sect. 3.1, the first phase of reconnection produces new closed loops and open right field. The magnetic field is ejected from the current sheet at Alfvénic velocities and, hence, may not settle into an immediate equilibrium. It is, therefore, not implausible to suggest that each reconnection reversal is driven by the rebounding of the reconnected field. However, we find no velocity signatures indicating a reversed motion of the field back towards the ends of the current sheet during the period after its creation and prior to the next $R_{2}$ reconnection phase commencing. Similarly, we find no reversed motion of any of the outflow fields during any of the subsequent reconnection phases and, thus, discount the possibility of oscillating loops or open field driving the oscillatory reconnection.

We continue to investigate why the reconnection ceases at the end of the first $R_{1}$ phase by considering the forces at work in the system at that time. Once ejected from the current sheet, the new closed loops collect in a confined region between the inflow magnetic fields and the photosphere, while the new open right field remains trapped between the open left and right field and the emerged loops. The continual addition of flux to these bounded outflow regions results in increasing levels of compression and, hence, increasing gas pressure there. The gas pressure gadient generated in the outflow regions is balanced by a Lorentz force and, thus, does not drive the first reconnection reversal. Similarly, we find that the forces in the centre of the inflow and outflow domains are balanced in all of the subsequent reconnection phases and, therefore, do not drive the reconnection reversals.

However, the compression within the outflow fields results in a significant contrast in the gas pressure across the boundaries or separatrices of the inflow and outflow regions that generates a gas pressure gradient acting perpendicular to the boundaries of the flux domains and towards the inflow regions, as shown in Fig. 6a. This force is counteracted by an opposing Lorentz force, again acting perpendicular to the boundaries but towards the outflow regions, as shown in Fig. 6d. The Lorentz force is generated by the combined force of the magnetic pressure gradient acting towards the magnetically depleted current sheet and the tension force pulling the curved fieldlines away from the current sheet. The tension force is marginally larger in the outflow regions where the newly reconnected fieldlines are sharply bent and, hence, the Lorentz force is directed towards the outflow regions. As will now be demonstrated, it is the balance between the gas pressure gradient and the Lorentz force at the boundaries of the inflow and outflow domains that controls the length of the current sheet, the maximum current in the current sheet and the reconnection rate.

In the latter stages of the first $R_{1}$ phase, the gas pressure gradient dominates over the Lorentz force and, thus, the inflow fields are pulled away from each other. The reason for this force imbalance will be explained shortly but the effects are two-fold. Firstly, at the ends of the current sheet, the pulling apart of the oppositely directed inflow fields results in the current sheet decreasing in length. Secondly, in the middle of the current sheet, the magnetic decompression associated with the pulling apart of the inflow fields decreases the current there and, thus, the reconnection rate decreases. Hence, we have a simultaneous decrease in the length of the current sheet, the maximum current in the current sheet and the reconnection rate, as discussed in Sect. 3.1.

The fact that the forces pulling the inflow fields apart are, consequently, bringing the outflow fields closer together is of key importance for the occurrence of a reconnection reversal. Once the inflow fields in the $R_{1}$ phase have been pulled apart to such an extent that reconnection has stopped and the current sheet has diffused away, the imbalance in the forces continues to carry the system past a simple X-point configuration and brings 

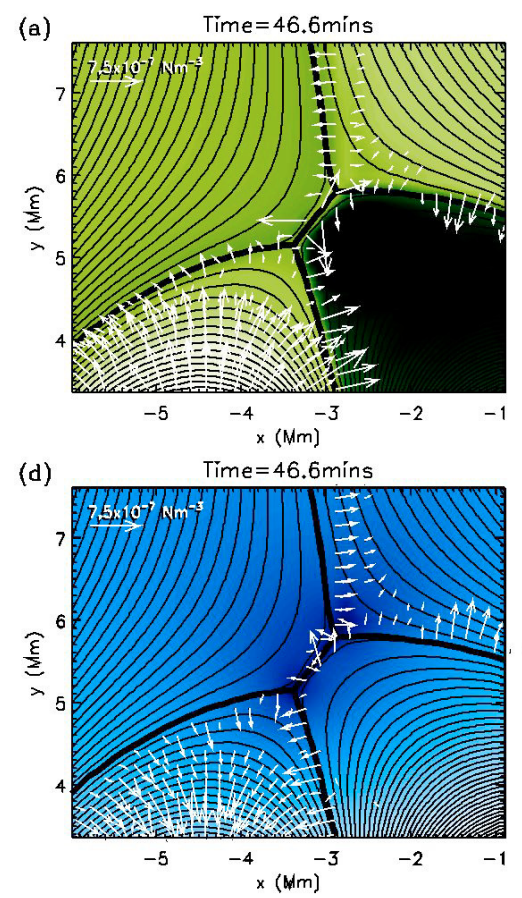
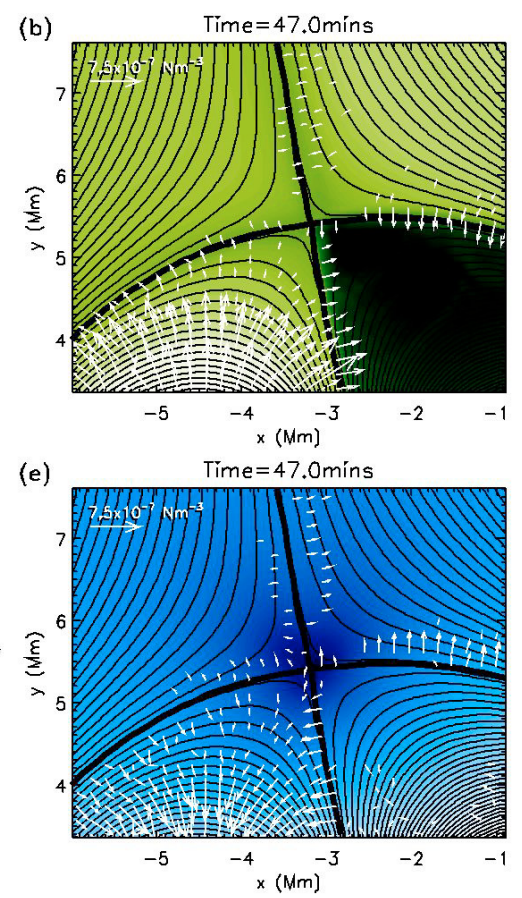
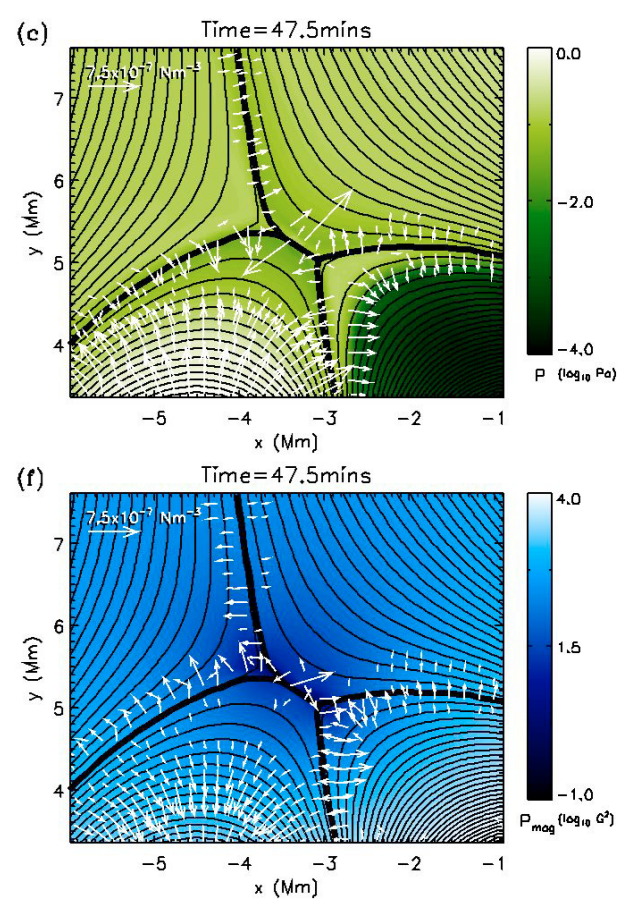

Fig. 6. a)-c) Contours of gas pressure, overlaid with a selection of fieldlines and arrows indicating the magnitude and direction of the gas pressure gradient, and $\mathbf{d}$ ) $-\mathbf{f}$ ) contours of magnetic pressure, overlaid with a selection of fieldlines and arrows indicating the magnitude and direction of the Lorentz force. The thick black lines indicate the boundaries or separatrices of the inflow and outflow regions. The period of time encompassed by the images covers the final stages of the first $R_{1}$ phase and the commencement of the following $R_{2}$ phase.

the outflow fields into contact. These outflow magnetic fields are oppositely directed and a current sheet develops. Reconnection sets in, marking the start of the first $R_{2}$ phase in which the outflow (inflow) fields of the $R_{1}$ phase become the inflow (outflow) fields in the $R_{2}$ phase.

As soon as reconnection commences, the ejections from the current sheet alter the properties of the new outflow regions. The high gas pressure and high tension force properties of the outflow fields reverse the direction of the forces acting at the boundaries of the regions, as shown in Figs. $6 \mathrm{c}$ and $\mathrm{f}$. The gas pressure gradient and Lorentz force still act towards the inflow and outflow regions, respectively, but the inflow and outflow regions in the current $R_{2}$ phase are reversed in comparison to the preceding $R_{1}$ phase. The tension force is immediately present but the gas pressure gradient increases slowly. Hence, during the early stages of the reconnection phase, the Lorentz force is larger than the gas pressure gradient and, thus, the inflow fields are pushed towards each other. Consequently, the current sheet increases in length and compression of the field towards the middle of the current sheet increases the maximum current there and the reconnection rate.

During the reconnection process, the gas pressure continues to increase in the outflow region and, thus, modifies the gradient across the boundaries of the domains. The magnitude of the tension force is maintained. Therefore, once the gas pressure gradient has increased enough to dominate the Lorentz force, the inflow fields are pulled apart. As in the $R_{1}$ phase, this firstly results in the length of the current sheet, maximum current in the current sheet and the reconnection rate decreasing and, secondly, brings the outflow fields closer together, preparing the way for the next reconnection reversal.

And so the oscillatory reconnection cycle continues, with the length of the current sheet, the maximum current in the current sheet and the reconnection rate increasing and decreasing in sync with each other as the imbalance in the gas pressure gradient and Lorentz force are modified.

From this description, we can see that increasing the gas pressure gradient at the boundaries of the domains is key to drawing the current reconnection phase to a close and bringing the outflow fields together, thus triggering a reconnection reversal. In order to increase the gas pressure gradient, compression must occur in the outflow regions and, therefore, the outflow fields must remain to some extent trapped and unable to escape far from the reconnection site. In this simulation, the photosphere, open field and new and emerged loops provide the bounds for the outflow regions during reconnection of types $R_{1}$ and $R_{2}$. This confinement condition is confirmed by the simulations of Chen \& Priest (2006), which fail to instigate a reversal when the reconnection between oppositely directed vertical fields is shut off by the growth of the outflow loop system. The field in the second outflow region is able to escape the numerical domain via the open top boundary in their experiments and, hence, no reconnection reversal occurs.

An equilibrium state for this system is one in which the forces at the boundaries of the domains are equal. However, the rapid reconnection carries the system past the equilibrium point during each phase of reconnection and, hence, the reconnection reversals occur. With each successive reconnection phase, the equilibrium is missed by a smaller margin and, thus, the imbalance in the forces pushing the inflow and outflow fields together is smaller. Consequently, the maximum current density achieved in the current sheet and the maximum length of the current sheet both decrease with each consecutive reconnection phase, shown in Fig. 5b. In line with this, the peak reconnection rate also decreases, as illustrated in Fig. 5a.

Reconnection halts after the last phase because the imbalance in the forces pushing the inflow and outflow regions together is negligible. Reconnection has taken the system as close to the equilibrium state as it can. Further progression of the 

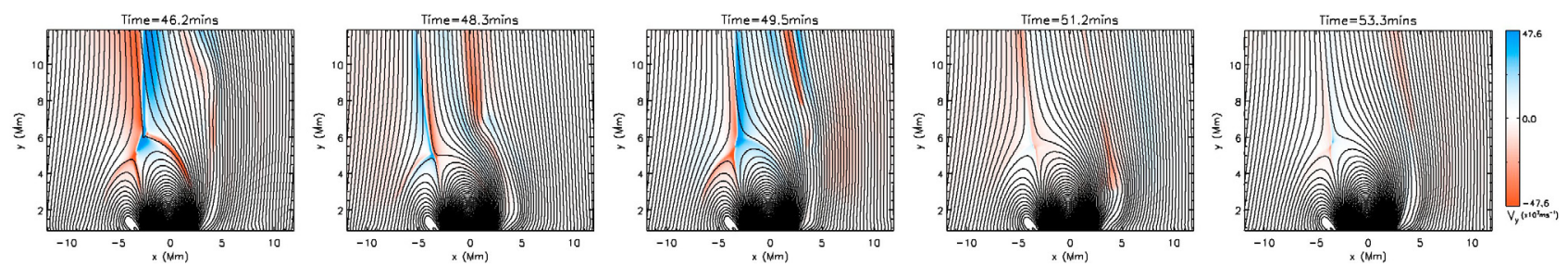

Fig. 7. Contours of vertical velocity, $v_{y}$, overlaid with a selection of fieldlines. The images correspond to times with reconnection of types $R_{1}$ or $R_{2}$, starting with $R_{1}$ and alternating from left to right.

system to the absolute equilibrium must now be achieved through small-scale oscillatory movements of the field.

To summarise, the mechanism of oscillatory reconnection is driven by the global imbalance of the forces between the neighbouring flux systems. The reconnection in this simulation probably occurs as a result of numerical rather than physical resistivity but, providing the reconnection proceeds sufficiently fast so that the system overshoots the equilibrium point, oscillatory reconnection is expected to set in under other resistivity models too. Modifying the resistivity model is anticipated to alter the value of the maximum reconnection rate and the period of the oscillations but the driving mechanism, and hence the main features of the model such as the synchronised growth and reduction of the maximum reconnection rate, maximum current and length of the current sheet, will remain unchanged.

\subsection{Consequences of oscillatory reconnection}

Although the general characteristics of each reconnection phase are the same, there are consequences of oscillatory reconnection that cannot be produced by a solitary reconnection phase.

The first consequence concerns the location of the outflow jets from the current sheet. Upon first leaving the current sheet, the jets travel into the outflow regions at angles perpendicular to the reconnected magnetic field they carry. Within seconds, the jets collide with the field already in the outflow regions and are deflected into two secondary jets travelling along the fieldlines and away from the collision site. Regardless of the type of reconnection occurring, each pair of outflow regions comprises closed loops and open field. Collision of the jets ejected from the lower end of the current sheet with the closed loops gives rise to flows with a negative $v_{y}$ velocity component as shown in Fig. 7. However, collision of the jets emitted from the upper end of the current sheet with the open field results in flows with a positive and negative $v_{y}$ velocity component along the same fieldlines. We refer to the positively directed part of this jet as a collimated jet since its trajectory remains vertical.

Of particular interest for comparison with observations is the collimated jet. By considering the central vertical axis of the collimated jet, we note that the jet will be displaced horizontally further from and closer to the centre of the current sheet as the latter grows and shrinks in length, respectively. During reconnection of type $R_{1}$ the displacement will be to the left of the current sheet but to the right during reconnection of type $R_{2}$. Together, these displacements make the collimated jet appear to sway during the oscillatory reconnection, as shown in Fig. 7.

The second consequence of oscillatory reconnection concerns the heated ejecta from the current sheet. The plasma in the current sheet is heated by ohmic dissipation and, subsequently, is carried away in the form of outflow jets. The reconnection reversals from $R_{1}$ to $R_{2}$ result in the open left field and emerged loop regions receiving hot outflow material, which would not have occurred under reconnection of type $R_{1}$ only. Additionally, the change in role of a region from being the outflow destination to the inflow location results in hot ejected field being pulled back into the current sheet during the next phase, as shown in Fig. 8. Hence, the oscillatory reconnection generates a cyclic temperature evolution.

In Sect. 3.2, we demonstrated that oscillatory reconnection ensues as the magnetic system settles towards an equilibrium state. At each subsequent reconnection reversal the system is closer to equilibrium and this has a number of effects on the outflow jets during consecutive phases. Firstly, the smaller maximum current in the current sheet results in less heating of the plasma there. Secondly, the rate at which the plasma is ejected from the current sheet reduces as the imbalance in the forces of the system decreases. Thus, in each subsequent reconnection phase the maximum temperature and maximum velocity achieved in the outflow jets decrease.

The inclusion of heat conduction in the simulations is expected to significantly reduce the temperature of the outflow jets, as heat is conducted along the fieldlines (Miyagoshi \& Yokoyama 2003, 2004). The density of the plasma ejections will also be affected by the addition of heat conduction, increasing to ensure a balance of forces in the current sheet is maintained, as detailed by Shiota et al. (2005). In the simulations of Shiota et al. (2005), the combined changes in temperature and density actually result in increased X-ray intensity and would make the outflowing hot plasma more easily detectable.

\section{Discussion}

The simulations we have performed clearly show oscillatory reconnection in action as a magnetic system searches for equilibrium after a flux emergence event. However, the simulations are carried out in 2.5 dimensions and represent simplified solar conditions. In this section, we will consider how the results would vary in a truly three-dimensional environment and whether there exists any observational evidence in support of oscillatory reconnection.

\subsection{Oscillatory reconnection in a $3 D$ environment}

In our simulations, oscillatory reconnection occurs because the outflows from the current sheet are ejected into bounded regions during every reconnection phase. A contrast builds between the properties of the inflow and outflow fields, creating forces acting at the boundaries of the inflow and outflow regions. Once the gas pressure gradient has grown large enough to dominate over the opposing Lorentz force, the current burst of reconnection is brought to a close and the next reconnection phase initiated.

In 2.5 dimensions, the whole length of an infinitely long flux tube is forced to rise as one body. Evolution is the same along the whole length of the tube and, therefore, expansion forces in 

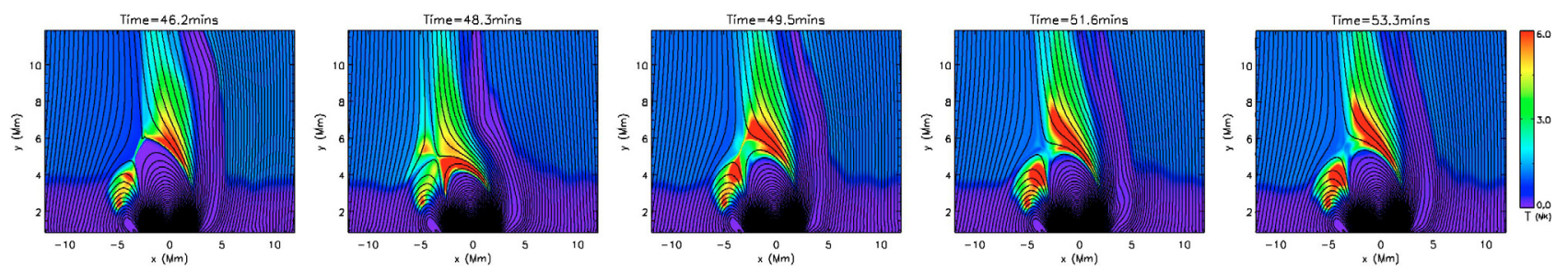

Fig. 8. Contours of temperature overlaid with a selection of fieldlines. The images correspond to times with reconnection of types $R_{1}$ or $R_{2}$, starting with $R_{1}$ and alternating from left to right.

the $z$ direction are exactly balanced in neighbouring $x-y$ crosssections of the tube. Hence, the interplay of the different forces is limited to the two-dimensional $x-y$ plane.

In a three-dimensional environment, a select portion of the tube's length can be made buoyant and, thus, the tube adopts an $\Omega$-shape during its rise to the surface. Expansion upon emergence becomes a fully three-dimensional process for the tube's magnetic field since not all of the neighbouring field is emerging at the same time and some parts do not emerge at all. Interchange reconnection will still be initiated between the emerged flux and the neighbouring coronal hole field, resulting in a new closed loop system and open field (Moreno-Insertis et al. 2008). The outflow regions will still be bounded in three dimensions so the setup is ripe for oscillatory reconnection.

As the first bout of reconnection progresses, the pressure will build in the outflow domains but any pressure differences can now be evened out in all directions. However, we believe that the pressure in the outflow system will contrast with that of the inflow regions and grow to a critical level where the inflow fields are pulled apart and, consequently, oscillatory reconnection will set in. The additional free direction means that it will take more time for the pressure to build in the outflow loop system and, thus, we expect reconnection phases will last longer in a threedimensional scenario.

\subsection{Supporting observational evidence}

Emerging flux has been identified as one of a number of triggers for coronal flares, which are rapid energy release events. Through a magnetic instability, such as reconnection, flares are believed to unlock the stored non-potential magnetic energy in magnetic fields. By releasing energy, a stressed magnetic system can return to a potential or lower energy equilibrium. Flares, therefore, are the perfect events in which to search for signs of oscillatory reconnection.

A number of flares on the Sun and stellar bodies have been found to display oscillatory emission behaviour which commences at or before flare maximum and continues into the decay phase (Asai et al. 2001; Mathioudakis et al. 2003, 2006; Mitra-Kraev et al. 2005; McAteer et al. 2005; Inglis et al. 2008). The oscillation period can measure tens or hundreds of seconds depending upon the individual event and have, in some instances, shown damping tendencies (McAteer et al. 2005; Mitra-Kraev et al. 2005; Inglis et al. 2008). Many of the authors have considered models of both bursty reconnection and periodic density enhancement to account for the intensity oscillations. Most have discounted the former as being unable to explain the damping characteristic and have concluded that an MHD mode must, therefore, be at work. Contrary to this, we find that oscillatory reconnection results in periodic changes in the maximum current and heating in the current sheet and that the largest maximum values in each phase reduce with time. Without accompanying evidence of oscillating motion, it is not possible to distinguish between the periodic reconnection and MHD mode models by damping oscillating intensity emission alone.

Periodic reconnection does not by itself constitute oscillatory reconnection. Our definition of oscillatory reconnection inherently requires a series of reconnection reversals, which consequently results in bursty or periodic reconnection. The reconnection scenario we have considered results in quasi-bounded outflow domains and it is this characteristic that ultimately enables oscillatory reconnection to occur. Fletcher et al. (2001) find an M1.9 solar flare occurring on 1999 May 3 is triggered from a site with an identical magnetic configuration to our simulations (see Fig. 11 of Fletcher et al. (2001)). In that case, the two domains of loops are initially in equilibrium but flux cancellation between the footpoints of one set of loops results in a loss of flux from this domain and the magnetic system as a whole is thrown out of equilibrium. Reconnection between the larger unaffected loop system and the surrounding field acts to rectify the inequality by taking flux from the larger domain and adding it to the smaller domain. Fletcher et al. (2001) model the magnetic field configuration of the flare event using a series of static potential field reconstructions. A technique such as this is highly likely to overlook any short-term dynamic events occurring and presents the idea that the equilibrium state is smoothly reached through a single phase of reconnection. However, in line with our results, it is reasonable to expect that the equilibrium state would be overshot during any phase of fast reconnection and equilibrium would be reached through oscillatory reconnection.

Changes in magnetic configuration as a result of a reconnection reversal have been observed by Goff et al. (2007), who considered a series of three solar flares. The final flare was a longduration event resulting from the relaxation of the magnetic field after the launch of a CME. The erupting and expanding flux rope results in forced reconnections between the CME and neighbouring magnetic fields. However, after the CME has left the region, the localised magnetic system relaxes into a natural equilibrium state through a reconnection reversal (see Fig. 12 of Goff et al. 2007). As in the model of Fletcher et al. (2001), we believe it is highly plausible that oscillatory reconnection will occur in order for the system to each a final equilibrium.

Finally, Sterling \& Moore (2001) and Sterling et al. (2001b,a) use isolated reconnection reversals to account for a series of homologous solar flares from a single active region. Each flare lasts $\sim 2.5 \mathrm{~h}$ and occurs over one complete cycle of three types of magnetic reconnection, two of which are analogous to $R_{1}$ and $R_{2}$. The cycle begins with new flux emerging between the field of a coronal hole and a sunspot (see Fig. 3 of Sterling \& Moore 2001). Reconnection between the legs of the emerged loops is identified as the source of the flare and creates an unrestrained flux rope and flare loops as by-products. Simultaneously, reconnection of type $R_{1}$ depletes the coronal hole field, establishing an escape path along which the flux rope 
leaves in the form of a CME. Following the CME, reconnection of type $R_{2}$ replenishes the coronal hole field and sets the system up such that the earlier reconnection processes can begin again. Three cycles of reconnection of types $R_{1}$ and $R_{2}$ are identified by the retreating and expanding boundary of the coronal hole in images from the extreme ultraviolet imaging telescope (EIT) aboard SOHO, the growth and shrinkage of "anemone" or new loops and the brightening and fading of EIT crinkles. The length of time between each flare and, hence, two successive reconnection reversals is $\sim 3 \mathrm{~h}$. In this particular scenario, the timescale is dependent on the rate of emergence of the new flux.

These observations illustrate that individual characteristics of oscillatory reconnection have been observed in association with flares. This is not to say that all flares should show signs of oscillatory reconnection since the occurrence of a flare is not restricted by magnetic topology to the same degree as the oscillatory reconnection mechanism. Additionally, oscillatory reconnection is not limited to flare events since emergence events can also create the required magnetic configuration, as shown by our simulations.

Given the driving mechanism of oscillatory reconnection, it can safely be assumed that this type of reconnection will occur at all scales. In both chromospheric and coronal observations, high speed swaying outflow jets have been seen above emitting loops (Cirtain et al. 2007; Shibata et al. 2007). These jets are similar to the upwards directed swaying outflow jets produced by oscillatory reconnection and are highly indicative of oscillatory reconnection occurring.

Finally, the heating of the different systems of loops as each becomes the outflow region may be identifiable in sequential spectral intensity images but no specific observations to date have indicated this feature.

In isolation, single characteristics are not definitive evidence of oscillatory reconnection occurring. Conclusive proof will require identification of multiple characteristics in a single observed event with a favourable magnetic configuration.

\section{Conclusions}

We have performed 2.5-dimensional MHD simulations of a flux rope emerging into the solar atmosphere endowed with a vertical magnetic field, which represents a coronal hole region. Previous studies have shown that this emergence scenario results in reconnection and, thus, we have used it to investigate the long-term evolution of a reconnecting magnetic system.

The results of our simulations in their early stages are well reported by other authors. A current sheet readily builds along the left-hand side interface between the expanding emerged loops and the neighbouring coronal hole field, where the magnetic fields are of opposite orientation. Interchange reconnection sets in and generates new closed loops and new open field with footpoints to the right of the emerged loops.

We find that oscillatory reconnection, a series of reconnection reversals, subsequently takes place. Oscillatory reconnection is defined by two main characteristics. Firstly, reconnection occurs in distinct bursts. Secondly, the inflow and outflow magnetic fields of one burst of reconnection become the outflow and inflow fields, respectively, in the following burst of reconnection. In isolation, this is termed a reconnection reversal and arises as a consequence of growing gas pressure in the quasi-bounded outflow regions. The gas pressure gradient acting across the boundaries of the inflow and outflow regions pulls the inflow fields apart, causing the current burst of reconnection to slow and stop, and brings the outflow fields together such that reconnection recommences. Over time, the system settles towards equilibrium as the imbalance in the forces across the boundaries of the inflow and outflow regions reduces. Once an equilibrium state is reached, all reconnection ceases.

Oscillatory reconnection in not a new phenomenon and has been studied using two-dimensional simulations (Craig \& McClymont 1991; McLaughlin et al. 2009). In these simulations, a wave is driven from the cylindrical boundary of a domain that contains a magnetic field of an X-type neutral point configuration. The propagating wave distorts the separatrices, boundaries separating the domains of different flux connectivity, and causes the central X-point to collapse. Oscillatory reconnection allows the stressed field to return to a state close to potential through changes in the magnetic topology. For Lundquist numbers smaller than $10^{4}$, Craig \& McClymont (1991) find that the decay time of the oscillations is shorter than the oscillation period and, thus, the reconnection oscillation is heavily damped. Our simulation has a coronal Lundquist number of $10^{6}$ and, hence, we find the oscillations damp reasonably quickly. Higher Lundquist numbers and, consequently, lower values for the resistivity should result in the oscillations continuing for longer and is likely to be more in line with oscillatory reconnection on the Sun. The testing of this assertion and appropriate parameter studies of other resistivity models are left for future research.

We believe that this paper is the first reported instance of oscillatory reconnection being initiated in a self-consistent rather than driven manner. It has offered a valuable insight into how oscillatory reconnection evolves in an everyday solar scenario and pinpointed the observable characteristics of the mechanism. Many of these characteristics have been observed in solar and stellar flares and solar atmospheric reconnection events. However, the characteristics of oscillatory reconnection and MHD modes are exceedingly similar and, in many observations, distinction between the two models is impossible.

Further numerical and observational investigations will be vital for increasing our understanding of oscillatory reconnection. The existence of oscillatory reconnection in a fully threedimensional environment is expected but must be verified. It is anticipated that the addition of heat conduction to the model will generate periodic heating at the footpoints of the loops in synchronisation with the periodic reconnection. This feature will then be readily comparable with emission oscillations of flare ribbons (McAteer et al. 2005). Observations searching for multiple characteristics of oscillatory reconnection in a single event will be key to proving the existence of oscillatory reconnection in real life plasma environments.

Acknowledgements. The authors would like to thank the referee, H. Isobe, for practical suggestions and perceptive comments that have enhanced this paper and A. W. Hood and J. A. McLaughlin for helpful discussions. The computational work for this paper was carried out on the joint STFC and SFC (SRIF) funded linux cluster at the University of St Andrews (Scotland, UK). M.J.M. and D.B. acknowledge financial assistance from the Science \& Technology Facilities Council (STFC) of the UK. L.v.D.G. acknowledges the Hungarian government grant OTKA T048961.

\section{References}

Arber, T. D., Longbottom, A. W., Gerrard, C. L., \& Milne, A. M. 2001, J. Computational Phys., 171, 151

Archontis, V., Moreno-Insertis, F., Galsgaard, K., Hood, A., \& O’Shea, E. 2004, A\&A, 426, 1047

Archontis, V., Moreno-Insertis, F., Galsgaard, K., \& Hood, A. W. 2005, ApJ, 635, 1299

Archontis, V., Galsgaard, K., Moreno-Insertis, F., \& Hood, A. W. 2006, ApJ, 645, L161 
Archontis, V., Hood, A. W. \& Brady, C. 2007, A\& A, 466, 367

Asai, A., Shimojo, M., Isobe, H., et al. 2001, ApJ, 562, L103

Baker, D., van Driel-Gesztelyi, L., \& Attrill, G. D. R. 2007, Astron. Nachr., 328, 773

Baker, D., van Driel-Gesztelyi, L., Murray, M. J., et al. 2009, Sol. Phys., submitted

Chen, P. F., \& Priest, E. R. 2006, Sol. Phys., 238, 313

Cirtain, J. W., Golub, L., Lundquist, L., et al. 2007, Science, 318, 1580

Craig, I. J. D., \& McClymont, A. N. 1991, ApJ, 371, L41

Crooker, N. U., Gosling, J. T., \& Kahler, S. W. 2002, JGR, 107, 1028

Fletcher, L., Metcalf, T. R., Alexander, D., Brown, D. S., \& Ryder, L. A. 2001 , ApJ, 554, 451

Galsgaard, K., Moreno-Insertis, F., Archontis, V., \& Hood, A. 2005, ApJ, 618, L153

Galsgaard, K., Archontis, V., Moreno-Insertis, F., \& Hood, A. W. 2007, ApJ, 666,516

Goff, C. P., van Driel-Gesztelyi, L., Démoulin, P., et al. 2007, Sol. Phys., 240, 283

Inglis, A. R., Nakariakov, V. M., \& Melnikov, V. F. 2008, A\&A, 487, 1147

Isobe, H., Miyagoshi, T., Shibata, K., \& Yokoyama, T. 2005, Nature, 434, 478

Isobe, H., Miyagoshi, T., Shibata, K., \& Yokoyama, T. 2006, PASJ, 58, 423

Leake, J. E., \& Arber, T. D. 2006, A\&A, 450, 805

Mathioudakis, M., Seiradakis, J. H., Williams, D. R., et al. 2003, A\&A, 403, 1101
Mathioudakis, M., Bloomfield, D. S., Jess, D. B., Dhillon, V. S., \& Marsh, T. R. 2006, A\&A, 456, 323

McAteer, R. T. J., Gallagher, P. T., Brown, D. S., et al. 2005, ApJ, 620, 1101

McLaughlin, J. A., De Moortel, I., Hood, A. W., \& Brady, C. S. 2009, A\&A, 493, 227

Mitra-Kraev, U., Harra, L. K., Williams, D. R., \& Kraev, E. 2005, A\&A, 436, 1041

Miyagoshi, T., \& Yokoyama, T. 2003, ApJ, 593, L133

Miyagoshi, T., \& Yokoyama, T. 2004, ApJ, 614, 1042

Moreno-Insertis, F., Galsgaard, K., \& Ugarte-Urra, I. 2008, ApJ, 673, L211

Murray, M. J., Hood, A. W., Moreno-Insertis, F., Galsgaard, K., \& Archontis, V. 2006, A\&A, 460, 909

Priest, E., \& Forbes, T. 2000, Magnetic reconnection: MHD theory and applications (Cambridge University Press)

Shibata, K., Nozawa, S., \& Matsumoto, R. 1992, PASJ, 44, 265

Shibata, K., Nakamura, T., Matsumoto, T., et al. 2007, Science, 318, 1591

Shiota, D., Isobe, H., Chen, P. F., et al. 2005, ApJ, 634, 663

Sterling, A. C., \& Moore, R. L. 2001, JGR, 106, 25227

Sterling, A. C., Moore, R. L., Qiu, J., \& Wang, H. 2001a, ApJ, 561, 1116

Sterling, A. C., Moore, R. L.. \& Thompson, B. J. 2001b, ApJ, 561, L219

Yokoyama, T., \& Shibata, K. 1995, Nature, 375, 42

Yokoyama, T., \& Shibata, K. 1996, PASJ, 48, 353

Zhang, J., Zhou, G., Wang, J., \& Wang, H. 2007, ApJ, 655, L113 